\title{
Fatal Acute Pancreatitis and Blood Alcohol Concentration. A Case Report and Literature Review
}

\author{
Mazzanti R*, Buscemi L, Valsecchi M and Tagliabracci A \\ Department of Forensic Pathology, Brazil
}

*Corresponding author: Mazzanti R, Department of forensic pathology, Brazil.

\begin{abstract}
Purpose
Design: Case report and literature review.

Methods: This was a case report of a 54-year-old woman, with an history of chronic alcohol abuse, who was found dead at home. A packet of trazodone was found next to the woman and an autopsy was performed for suspected suicide. Literature regarding the epidemiology and social characteristics was searched from the PubMed database.

Conclusion: Fatal acute pancreatitis occurring outside of the hospital concerns many patients who lived alone and in poor social conditions, and alcohol abuse is a common factor, also in the cause of death.
\end{abstract}

Keywords: Acute pancreatitis; Sudden death, Blood alcohol concentration; Forensic pathology

\section{Introduction}

Acute pancreatitis represents a spectrum of disease, ranging from a mild, self-limited disorder, consisting of acute interstitial inflammation and oedema, with only slight or no acinar cell necrosis, to a severe, rapidly progressive and sometimes fatal hemorrhagic form, with massive necrosis [1]. The incidence of acute pancreatitis varies considerably between geographic regions and is estimated at 5-80 per 100.000 population. The overall mortality rate of acute edematous pancreatitis is below $1 \%$, but when considering the severe hemorrhagic form of the disease, mortality rates range from $13.5-24 \%$ [2]. While a large number of studies have dealt with the clinical picture, course, and outcome of acute pancreatitis in the clinical setting, post-mortem studies of fatal acute pancreatitis are sparse. Especially individual characteristics of those outpatient fatalities that begin with an abrupt onset and soon after presents as sudden, unexpected death and of those cases where death occurs immediately after admission to hospital seem to be largely unknown. Investigation of sudden and unexpected death is a challenge for a forensic pathologist. The definition of sudden death varies according to authority and convention. The World Health Organization defines sudden death as those occurring within $24 \mathrm{~h}$ following onset of symptoms, including all instantaneous deaths, and all deaths occurring within $24 \mathrm{~h}$ of an acute collapse. Most of sudden death occur outside the hospital or in emergency rooms, reflecting their unexpected nature. In deaths that are both sudden and unexpected; non-availability of clinical data and non-existing physical signs, makes the job of the forensic experts tougher.

\section{Case Information}

The deceased was a woman aged 54 years, moderately built and nourished. A packet of trazodone was found next to the woman, who had in the past tried to commit suicide by ingesting psychotropic drugs. No external injuries were present on the body, there were residues of food vomiting. On internal examination, heart appeared normal and weighed 300g. Brain was edematous and weighed 1230g; left and right lungs were edematous and weighed $470 \mathrm{~g}$ e $380 \mathrm{~g}$ respectively. The trachea and esophagus were normal. Stomach was empty. Spleen, liver and both kidneys were congested (Figure 1). Pancreas was soft, pulpy, friable and edematous, with areas of frank hemorrhages. There was no evidence of gallstones, infection or obstruction of the biliar ducts. The organs were subjected to histopathological examination. Tissue specimens from pancreas, liver, heart, adrenal, kidney and brain were fixed in $10 \%$ formalin, embedded in paraffin and stained with hematoxylin and eosin for histological studies (Figure2). 


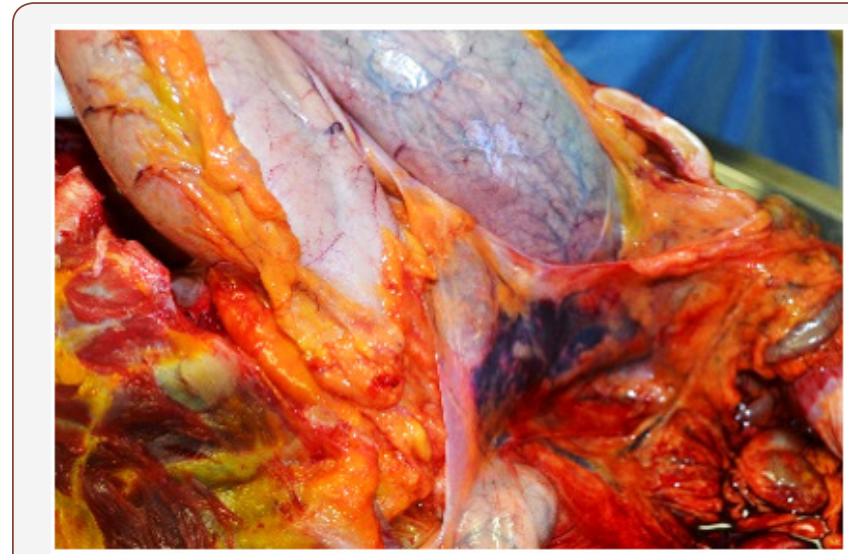

Figure 1: Examination of the abdominal cavity (cavity of epiploon).

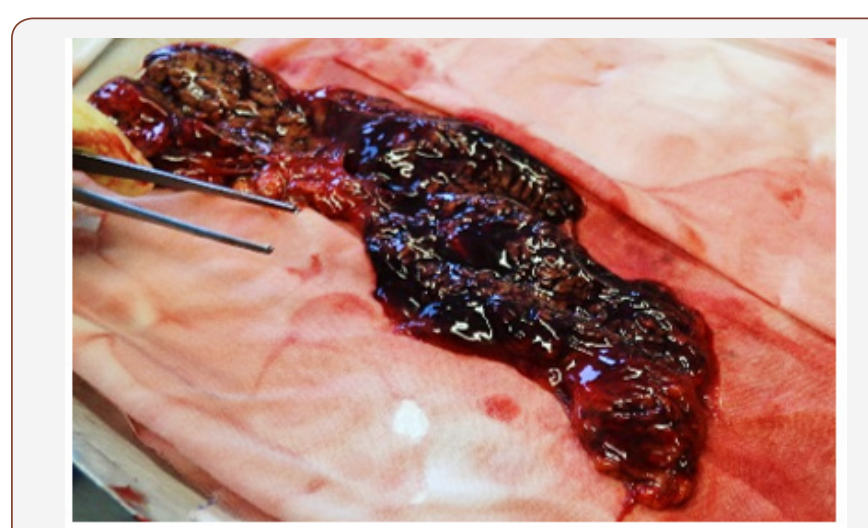

Figure 2: Gross view of the pancreas showing a soft, pulpy, oedematous appearance and massive haemorrhagic necrosis of pancreatic tissue along its head, body, and tail.

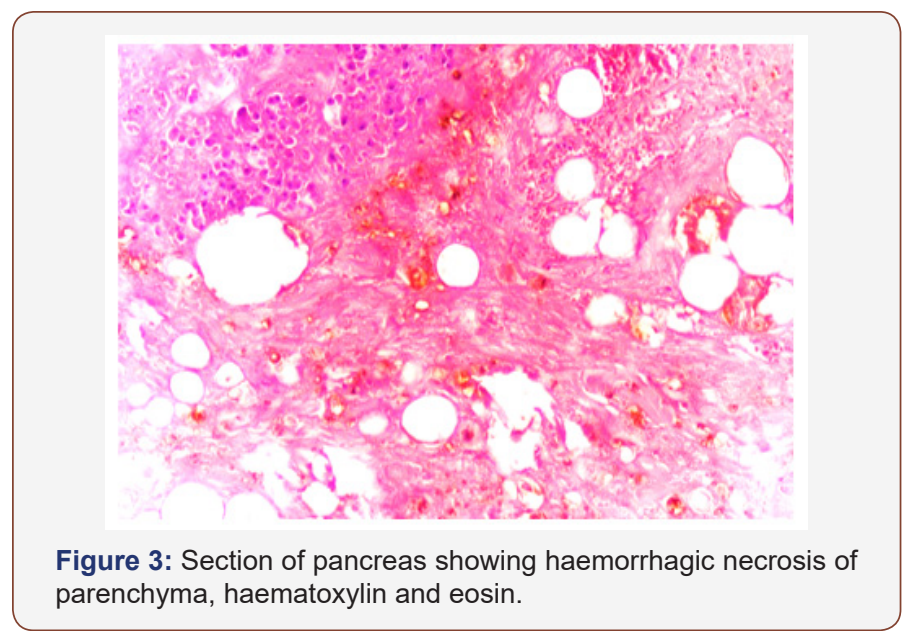

Sections of pancreas showed total hemorrhagic necrosis of the pancreatic parenchyma including acini and islets, which are indicative of acute hemorrhagic pancreatitis. Sections from the kidneys showed intense congestion. Sections from the lungs showed diffuse alveolar damage with intense congestion of capillaries. Toxicological screening of blood was positive for alcohol $(2.14 \mathrm{~g} / \mathrm{L})$, isolated by head-space technique, and negative for drugs and poisons. The cause of death was attributed to acute cardio-respiratory failure to severe acute hemorrhagic-necrotizing pancreatitis in a state of acute alcohol intoxication after a detailed post-mortem examination, histological study of tissues, and toxicological analysis (Figure3).

\section{Discussion}

Acute pancreatitis is a condition associated with acute and sudden inflammation of the pancreas that may involve the peripancreatic tissue and various organ system. In majority of patients, acute pancreatitis is a mild disease, usually associated with a rapid recovery within a few days of onset of the illness. Gallstones and excessive alcohol usage are the most common causes for injury to the pancreas and account for more than $85 \%$ of all patients that develop pancreatitis [3]. Hyperlipidemia, hypocalcemia, many drugs, and blunt trauma to the abdomen have been associated with pancreatitis. The most frequent symptom in acute and recurrent pancreatitis is abdominal pain, following by vomiting. The major symptom, abdominal pain may vary widely in intensity from the typical sharp incapacitating pain to mild bearable pain with more or less asymptomatic onset. In about $20 \%$ of the patients with acute pancreatitis, sever damage to the pancreas may lead to a lifethreatening illness. Early deaths are usually due to a multisystem organ failure. Inflammatory mediators and cytokines originating from the inflamed pancreas may be responsible for damage to vital organs by mechanisms such as vascular injury, stasis, or enhanced intravascular coagulation. These deaths are usually manifested by irreversible pulmonary edema and acute renal failure. In acute pancreatitis, the most common extra-pancreatic pathology has been reported in the lung. The prevalence of pulmonary complications in autopsy studies ranges from $20 \%$ to $100 \%$, pulmonary edema being the most common finding [4]. Cases of sudden death due to acute pancreatitis have been reported in the past where diagnosis could not be made until autopsy [5-7]. Based on the current literature, we analyses the social characteristic, with special regard on history of alcohol consumption.

In a retrospective study twenty-seven cases of acute pancreatitis that presented as sudden, unexpected death were identified and included. Based on information obtained from family members and witness reports from police files, a history of chronic alcohol consumption could be established in 16 cases (59\%). According to the available official reports, at least 20 subjects had lived socially isolated, with no contact to family or neighbors, or had belonged to social fringe groups (e.g. homeless people). Toxicological analysis was positive for alcohol in femoral venous blood obtained at autopsy in 4 case (BACs, $7-141 \mathrm{mg} / \mathrm{dL}$ ) [8].

In another retrospective study, they found that based on information in police files, including witness reports and documentation on living conditions, a history of chronic alcohol consummation could be established in all but one of the cases where alcohol was suspected to be the etiological factor. The remaining subject was a woman with high blood alcohol concentration at autopsy. Toxicological analyses were performed, and 13 patients had an elevated level of ethanol in femoral blood [9]. In a study performed in Northern Ireland, they included 27 deaths from pancreatitis in which the diagnosis was made only at post-mortem examination. Of the undiagnosed fatalities, 10 occurred in individual with a history of alcohol abuse [10] (Table 1). 
Table 1: Cases of high BAC at time of death.

\begin{tabular}{|c|c|c|c|}
\hline & $\begin{array}{c}\text { Reported } \\
\text { Cases }\end{array}$ & $\begin{array}{c}\text { History of } \\
\text { Alcohol Abuse }\end{array}$ & $\begin{array}{c}\text { Bacs at Time of } \\
\text { Death }\end{array}$ \\
\hline Tsokos M et al. [8] & 27 & $16(59 \%)$ & $\begin{array}{c}4(14 \%)[0.07- \\
1.41 \mathrm{~g} / \mathrm{L}]\end{array}$ \\
\hline Andersson B et al. [9] & 50 & $49(98 \%)$ & $\begin{array}{c}13(26 \%)[1.32- \\
1.66 \mathrm{~g} / \mathrm{L}]\end{array}$ \\
\hline Heatley MK et al. [10] & 27 & $10(37 \%)$ & - \\
\hline $\begin{array}{c}\text { Andersson R et al. } \\
\text { [11] }\end{array}$ & 36 & $27(75 \%)$ & $\begin{array}{c}15(42 \%)[0.2- \\
4.1 \mathrm{~g} / \mathrm{L}]\end{array}$ \\
\hline
\end{tabular}

A similar outcome came from a Swedish study in which in 15 out of 27 patient's dead from fatal pancreatitis, measurable ethanol concentrations were demonstrable in cardiac blood, with in median $0.8 \mathrm{~g} / \mathrm{L}(0.2-4.1 \mathrm{~g} / \mathrm{L})[11]$. In general, we can say that the majority of the patient were found in their own home. Many of the persons lived alone, and sometimes they were homeless. In our case, the woman lived alone, and she had an history of chronic alcohol abuse and survived a suicide attempt during the last year. A packet of trazodone was found next to the woman, but the toxicological screening of blood was negative for drugs and poisons.

As reported in the study by Tosco's and Braun, in which 74\% of the patients lived isolated with no social contacts, it is the lifestyle that makes these patients vulnerable. Investigation of the patients' social network, life, and circumstances of death in acute pancreatitis has not previously been performed. A study by Ellis et al [12] accounts for, to the best of our knowledge, the only previous publication touching this issue. In a comprehensive epidemiological study of acute pancreatitis, they found a clear relationship between socioeconomic deprivation and incidence of the disease, which was largely explained by a higher incidence of alcoholic etiology.

\section{Conclusion}

Patients who die from acute pancreatitis who were never admitted to the hospital, as in our case, have some characteristics in common. Alcohol abuse is frequent, and sometimes the blood alcohol concentration at the time of death is high. An elevated alcohol concentration may lead the subject to underestimate the symptoms - it may be that the patient 'treats' first symptoms at the onset of acute pancreatitis with intake of more alcohol - to produce important effects on the state of consciousness (comatose or so porous state) and, obviously, to favor a depression of the respiratory centers of the brainstem, leading to a fast and sudden death. We can conclude that this is an important implication the forensic pathologist that investigate on a sudden death of an isolated person, who have an history of alcohol abuse.

\section{Acknowledgement}

None.

\section{Conflict of Interest}

No conflict of interest.

\section{References}

1. Nathens AB, Curtis JR, Beale RJ, Cook DJ, Moreno RP, et al. (2004) Management of the critically ill patient with severe acute pancreatitis. Crit Care Med 32(12): 2524-2536.

2. Mayerle J, Hlouschek V, Lerch MM (2005) Current management of acute pancreatitis. Nat Clin Pract Gastroenterol Hepatol 2(10): 473-483.

3. Steinberg W, Tenner S (1994) Acute Pancreatitis. N Eng J Med 330: 11981210.

4. Kanchan T, Shetty M, Nagesh KR, Khadilkar U, Shetty BS, et al. (2009) Acute haemorrhagic pancreatitis-A case of sudden death. J For and Legal Med 16(2): 101-103.

5. Dalgaard JB (1956) Pancreatitis as the cause of sudden death in alcoholics. Acta Pathol Microbiol Scand 39(3): 185-194.

6. Toffler AH, Spiro HM (1962) Shock or coma and the predominant manifestation of painless acute pancreatitis. Ann Intern Med 57: 655659.

7. Lankisch PG, Rahlf G, Koop H (1983) Pulmonary complications in fatal acute haemorrhagic pancreatitis. Dig Dis Sci 28(2): 110-116.

8. Tsokos M, Braun C (2007) Acute Pancreatitis Presenting as Sudden, Unexpected Death. An autopsy-based Study of 27 Cases. Am J Forensic Med Pathol 28(3): 267-270.

9. Andersson B, Ansari D, Andersson E, Persson S, Andersson R (2010) Fatal Acute Pancreatitis Occurring Outside of the Hospital: Clinical and Social Characteristics. World J Surgery 34(10): 2286-2291.

10. Heatley MK, Crane J (1989) Acute pancreatitis as a cause of sudden or unexpected death in Northern Ireland. The Ulster Med J 58(1): 51-55.

11. Andersson R, Andrén-Sandberg A (2003) Fatal Acute Pancreatitis. Characteristics of Patients Never Reaching Hospital. Pancreatology 3(1): 64-66.

12. Ellis MP, French JJ, Charnley RM (2009) Acute pancreatitis and the influence of socioeconomic deprivation. Br J Surg 96(1): 74-80. 